INFN - Istitute Nazionale di Fisica Nucleare

Sezione di Perugia

INFN/AE-93/22

4 Novembre 1993

\title{
CORRELATION BETWEEN FUTURE AND PAST PHOTON EVENTS
}

A. Widom, Y. N. Srivastava and E. Sassaroli

Physics Department \& INFN, University of Perugia, Perugia, Italy

and

Physics Department, Northeastern University, Boston, Massachusetts, USA

\begin{abstract}
The notion that future events can affect present events was discussed by Einstein, Tolman and Podolsky as an intrinsic part of the quantum mechanical theory. It is here shown that a two photon experimental test of this prediction is well within the present technology of quantum optics.
\end{abstract}




\section{Introduction}

In the well known debates between Einstein and Bohr on the meaning of quantum mechanics, Bohr emerged victorious in that the predictions of quantum mechanics (although often counter to expectation) worked well in all known laboratory experiments. Among the least intuitive of Einstein's notions was the dual nature of quantum uncertainties, and the propagation of such uncertainties in both forward and backward directions in time. That uncertainties in future events could be influenced by present events seemed more or less natural. That uncertainties in present events could be influenced by future events seemed (at first glance) far less likely. The above "quantum telepathic" notion ${ }^{(1)}$ was nevertheless the subject of a remarkable note by Einstein, Tolman and Podolsky ${ }^{(2)}$, and will be here called the ETP effect. Both Bohr and Heisenberg agreed with the ETP effect as a genuine prediction of quantum mechanics ${ }^{(3)}$. They did not regard the apparent "lack of causality" as in any way a defect in the quantum theory.

The notion of signals moving in both time directions (as an intrinsic feature of relativistic quantum mechanics) was invoked somewhat later by Stückelberg ${ }^{(4)}$ in his description of the positron as an electron moving backward in time. Feynman ${ }^{(5)}$ built on this notion of "anti-particles" as "particles traveling backward in time" to formulate a complete diagrammatic calculation technique, now standard in relativistic quantum mechanics. However, the physical roots of the subject (in the ETP effect for two photons) are often lost in a forest of mathematical trees and multiloops. In what follows, we hope that the simplicity of the mathematical presentation will clarify the physics of the ETP effect. We note (in passing) that Einstein, Tolman and Podolsky wrote down no equations at all.

\section{Two Photon States}

Shown in Figure 1 is a well known optical setup for measuring the Transmission $T_{\alpha}$ and reflection $R_{\alpha}$ probability of a photon $\alpha$ incident on a partially reflecting plate. The amplitudes for an incident photon to arrive at detectors $i=1,2$ are denoted by $\alpha_{i}$. It is assumed that the plate has virtually no photon absorption, i.e., the reflection probability to arrive at detector 1 and the transmission probability to arrive at detector 2 sum to unity,

$$
R_{\alpha}+T_{\alpha}=\left|\alpha_{1}\right|^{2}+\left|\alpha_{2}\right|^{2}=1
$$

Similar considerations would apply to another photon $\beta$,

$$
R_{\beta}+T_{\beta}=\left|\beta_{1}\right|^{2}+\left|\beta_{2}\right|^{2}=1
$$

It is important for the later consideration of two photon wave functions that the single photons $\alpha$ and $\beta$ be neither completely the same nor be totally different, i.e., that the single photon wave functions should have a finite "overlap" $\epsilon$,

$$
\epsilon=\alpha_{1}^{*} \beta_{1}+\alpha_{2}^{*} \beta_{2} \text {. }
$$

Let us now review the nature of the two photon ETP effect, and consider the normalized and properly Bose symmetrized two photon wave-function

$$
\Psi_{i j}=\frac{\alpha_{i} \beta_{j}+\alpha_{j} \beta_{i}}{\sqrt{2\left(1+|\epsilon|^{2}\right)}}
$$

With two photons incident on the partially reflecting plate, we may compute (from the wave function) the mean number of photons reaching each detector as well as the correlation between two photon arrivals for each trial. For example, the mean number of photons arriving at detector 1 is given by

$$
<N_{1}>=2\left|\Psi_{11}\right|^{2}+\left|\Psi_{12}\right|^{2}+\left|\Psi_{21}\right|^{2}
$$


The correlation between one photon arriving at detector 1 and one photon arriving at detector 2 is given by

$$
<N_{1} N_{2}>=\left|\Psi_{12}\right|^{2}+\left|\Psi_{21}\right|^{2}
$$

Photon arrivals and correlation have internal consistency relations, e.g., the number of ETP photons is in total two for each experimental trial. An example of such a consistency relation follows from Eqs.(1) -(6);

$$
<N_{1}>=\frac{2 R_{\alpha} R_{\beta}+\left(1-R_{\alpha}-R_{\beta}\right)<N_{1} N_{2}>}{2 R_{\alpha} R_{\beta}+\left(1-R_{\alpha}-R_{\beta}\right)}
$$

$\mathrm{Eq}(7)$ is central to what follows.

\section{Time Delay and the ETP Effect}

In Figure 2 we show a two photon process scattering off a partially reflecting plate. If the photons have a reasonably well defined frequency difference $\Delta \omega=\omega_{\alpha}-\omega_{\beta}$ and a time delay $\tau$ is constructed before detector 2 , then $\tau$ will have an effect on the amplitudes of arrival at the two detectors of the photons. For example eqs.(1),(2) and (3) imply that

$$
|\epsilon|^{2}=R_{\alpha} R_{\beta}+T_{\alpha} T_{\beta}+2 \sqrt{T_{\alpha} T_{\beta} R_{\alpha} R_{\beta}} \cos (\phi+\tau \Delta \omega)
$$

From considerations such as these, one obtains a standard correlation expression for the manner in which two photon arrival at detectors (one photon at detector 1 and one photon at detector 2) varies with the time delay $\tau$; e.g., Eqs.(4) and (6) now read

$$
<N_{1} N_{2}>=\frac{1-R_{\alpha} R_{\beta}-T_{\alpha} T_{\beta}+2 \sqrt{T_{\alpha} T_{\beta} \bar{R}_{\alpha} \bar{R}_{\beta}} \cos (\phi+\tau \Delta \omega)}{1+R_{\alpha} R_{\beta}+T_{\alpha} T_{\beta}+2 \sqrt{T_{\alpha} T_{\beta} R_{\alpha} \bar{R}_{\beta}} \cos (\phi+\tau \Delta \omega)}
$$

Eq.(9) for photon arrival correlation is both theoretically and experimentally valid. Quantum optics has reached the stage for which photon arrival correlation and coherence are well understood ${ }^{(6)}$.

The crucial point is that the intensity correlation between the detectors (here described by $<N_{1} N_{2}>$ ) is an experimental function of the time delay $\tau$. But from Eq.(7), it then follows that the mean number of photons $\left\langle N_{1}\right\rangle$ (per two photon scattering trial) arriving at detector 1 depends on the imposed time delay $\tau$ to detector 2. Thus, the later detection can influence the earlier detection. This ETP effect is plotted in Figure (3).

\section{Conclusions}

How can we "explain" that the probability of an early photon detection at detector 1 depends on a time delay $\tau$ to detector 2 where another photon (perhaps) will later arrive?. Beyond the fact that quantum mechanics predicts this ETP effect, it is certainty not very intuitive. (A closely analogous ETP effect for the two $\mathrm{K}$ meson decay of the $\Phi$ resonance has been previously discussed by the authors ${ }^{(7)}$.)

From the viewpoint of quantum field theory, future events having an effect on present events is quite satisfactory. An antiparticle, born in the future, travels backward in time and can be annihilated in the present. (Possible death before birth is a more or less obvious hazard of time travel). For the photon, the particle and the anti-particle are very much the same sort of particle.

Unlike the electron and the positron, there is no "sign of the charge" to distinguish the direction in time of the photon path. A photon, moving backward in time and then changing its derection to move forward in time, would appear to a laboratory observer as two photon creation event. Such is the nature of the Feynman 
diagramatic rules in quantum electrodynamics. Those who would invoke the word "virtual" (to avoid any claim of "physical reality" to the "paths in time" for quantum electrodynamic amplitudes) should enjoy Feynman's derivation of his own rules. Feynman argued ${ }^{(8)}$ that all emitted and then absorbed photons may be treated as "virtual".

As always, it is the experimentalist who ultimately decides what is "virtual" and what is "real". The "physical reality" of the two photon ETP effect can only be tested in the laboratory. While many measurements of delay time variations of the "early-late" correlation $\left\langle N_{1} N_{2}\right\rangle$ exist, there have been no reported measurements of $\tau$ variations in the "early" arrival $\left\langle\mathrm{N}_{1}\right\rangle$. Such data are required to investigate the ETP effect.

\section{References}

1. A. Einstein in "Albert Einstein Philosopher Scientist", p. 168, Library of Living Philosophers, P. A. Schlipp, Ed., Open Court Publishing Co. Illinois (1970).

2. A. Einstein, R. Tolman, and B. Podolsky, Phys. Rev. 37, 780 (1931).

3. A. Fine, "The Shaky Game: Einstein Realism and the Quantum Theory", p. 31, University of Chicago Press, Chicago (1986).

4. E. C. C. Stückelberg, Helv. Phys. Acta 15, 23 (1942).

5. R. P. Feynman, Phys. Rev. 74, 993 (1948); Ibid. 76, 769 (1949).

6. For a recent survey see for example, "Quantum Measurements in Optics", P. Tombesi and D. Walls, Eds., Plenum Press, New York (1992).

7. Y. N. Srivastava and A. Widom, Phys. Lett. B314, 315 (1993).

8. R. P. Feynman, Phys. Rev. 80, 440 (1950), Appendix B. 


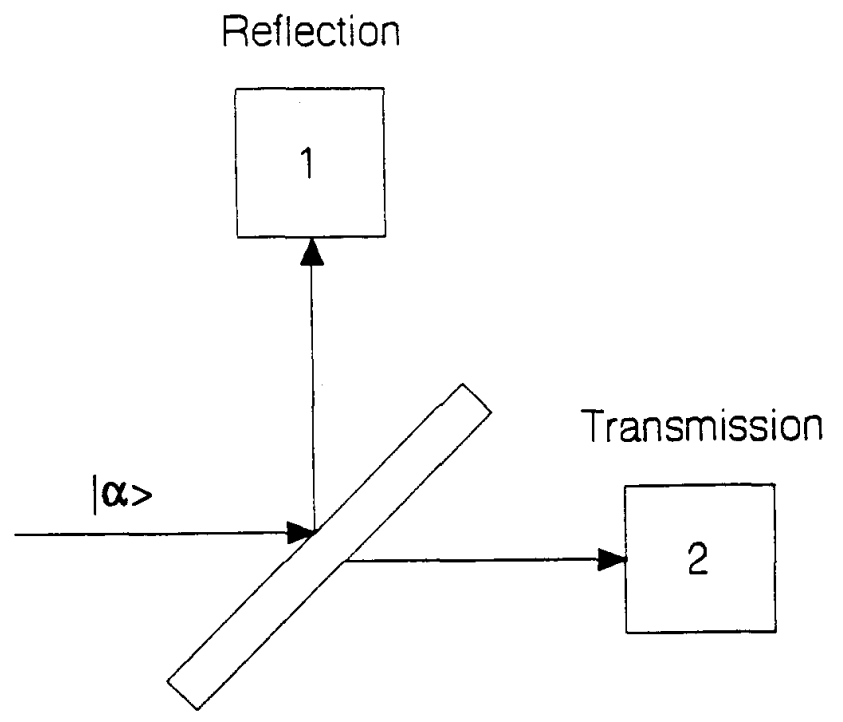

FIG. 1- Conventional experimental arrangement for measuring the reflection probability $R_{\alpha}$ and transmission probability $T_{\alpha}$ for a photon $\alpha$ incident on a partially reflecting plate.

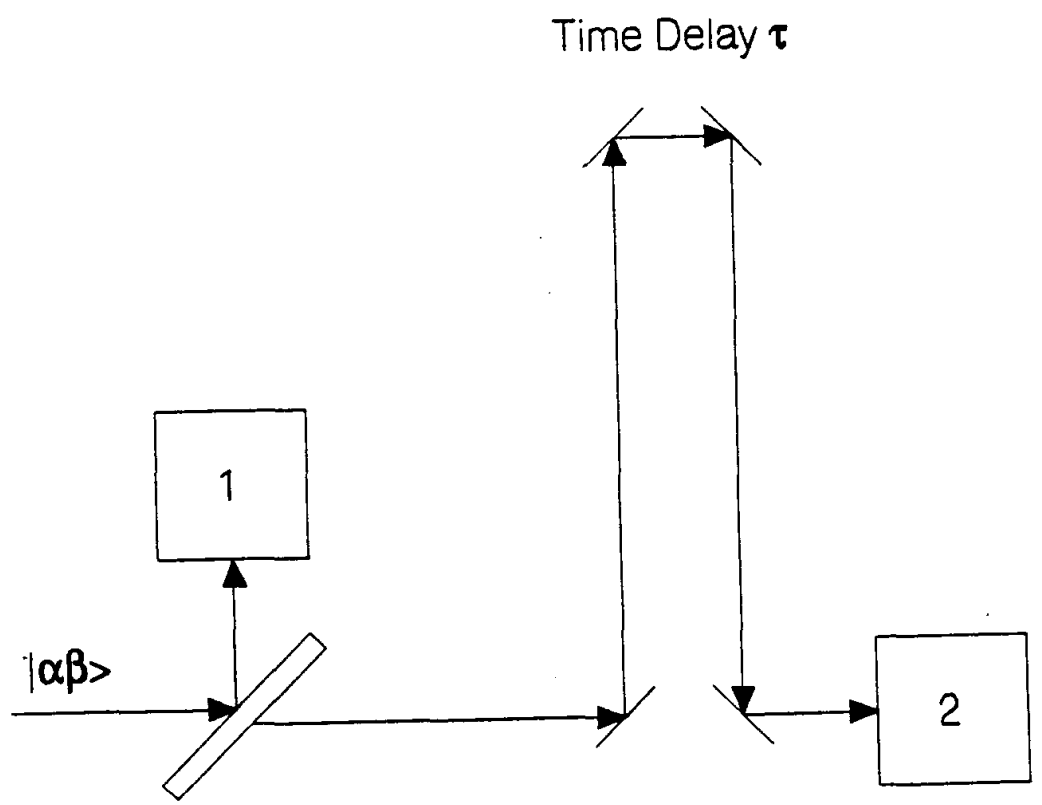

FIG. 2 - With two photons $\alpha$ and $\beta$ incident on a partially reflecting plate, the mean number of photons (per trial) $\left\langle\mathrm{N}_{1}>\right.$ at detector 1 will depend on a "future time delay $\tau$ " to the detector 2 . 


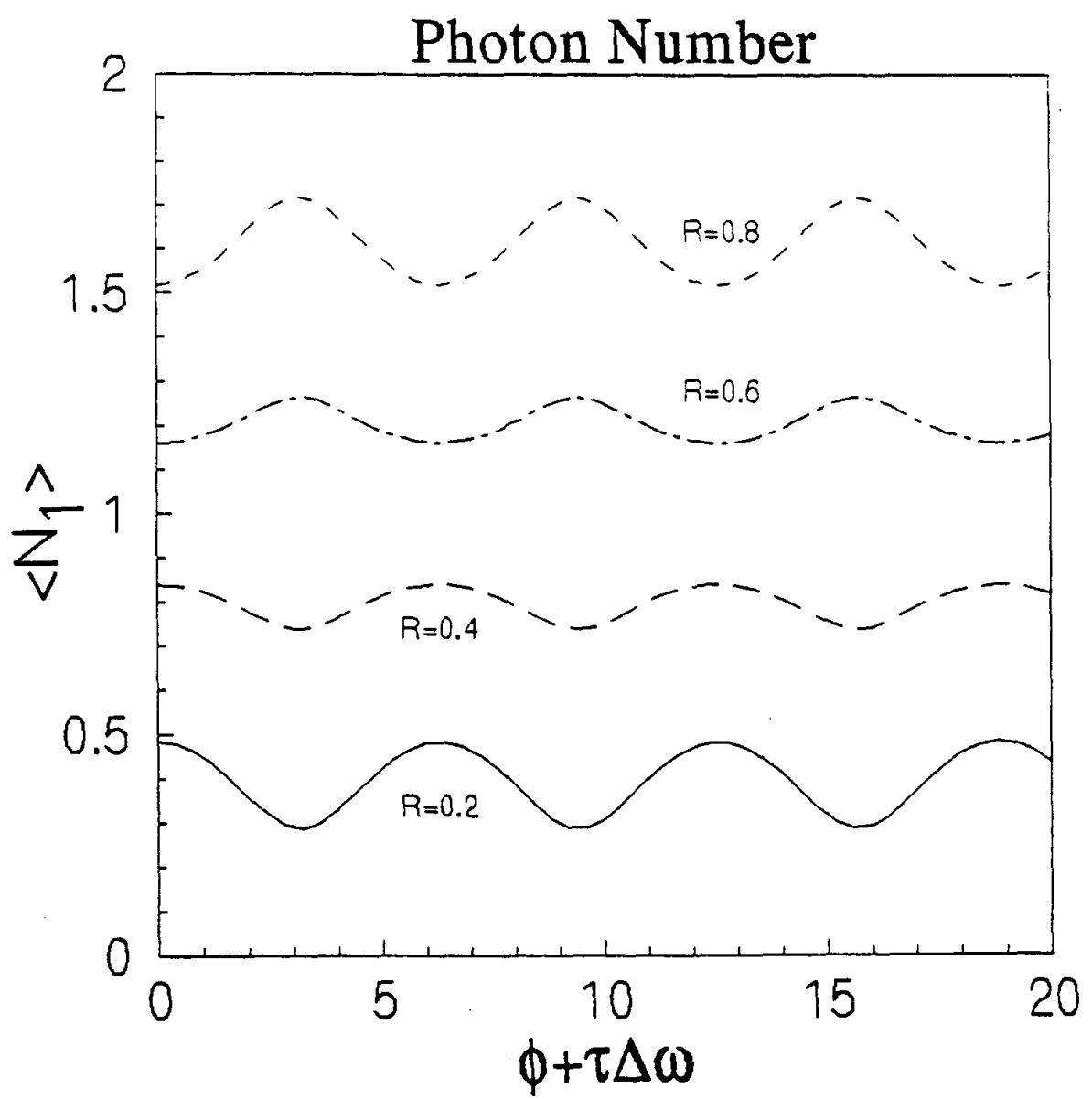

FIG. 3 - Plot of $\left\langle N_{1}>\right.$ versus the phase delay $(\phi+\tau \Delta \omega)$ for the experimental arrangement of Fig. 2. For illustrative purposes we assume $R_{\alpha}=R_{\beta}=R$, and exhibit the variations for $R=0.2,0.4,0.6$ and 0.8 . 